\title{
Topology Optimization of a Piezoelectric Energy Harvester
}

Wein, Fabian; Weller, Elias; Albach, Thorsten; Sutor, Alexander; Lerch, Reinhard

Department for Sensor Technology, University Erlangen-Nuremberg

Paul-Gordan-Str. 3/5, 91052 Erlangen, Germany

\begin{abstract}
We use a thin piezoelectric layer attached to a mechanical plate to harvest mechanical energy as available at the vibrating skin of machinery. By modifying the material distribution of the piezoelectric layer the resonance frequency of the energy harvester is adjusted to maximize the objective function. In this paper the electric energy and the electric potential is maximized. This topology optimization problem is performed by a variant of the SIMP (Solid Isotropic Material with Penalization) method which determines for every finite element cell if it is better to have piezoelectric material at that location or not. Numerical results are shown for single frequency pressure excitations from a broad frequency range. The objective functions electric potential, electric energy, energy conversion factor and mean transduction are discussed.
\end{abstract}

\section{Introduction}

Energy harvesters are devices producing electric energy out of ambient energy. The applications and concepts are of wide range. Typical applications are self powered sensors with wireless communication. This might allow for more flexibility in the location of the devices, would be cost efficient as cables or batteries are not required and environmental friendly as less batteries have to be produced.

Devices built of piezoelectric material harvesting vibrational ambient energy represent an area of active research. Due to the typical small power output, an increase in performance or reduction of cost has a significant impact. A comprehensive overview is given in the review paper (Anton and Sodano, 2007), where several optimization efforts are mentioned but none applying topology optimization.

In Sections 2 and 3 we introduce our model for the finite element formulation and introduce and apply the SIMP (Solid Isotropic Material with Penalization) method.

To our knowledge there are only two publications about the topology optimization of energy harvesters (Nakasone et al, 2008) and (Bin Zheng and Gea, 2008). They are discussed in Section 4 along our own objective functions (potential and electric energy).

Numerical results and conclusions finish this work. Due to the complexity of the subject and limited space we refer to our publications (Wein et al, 2008a) and (Wein et al, 2008b) giving a more comprehensive introduction to a similar coupled model (but there as actuator).

\section{The Composite Model}

In contrast to beam based energy harvesters, we consider thin plates or films subject to deformation due to mechanical pressure. This pressure might result from acoustic pressure, fluid or mechanical excitation. As depicted in Figure 1(a) a layer of piezoelectric material $\Omega_{p}$ is attached to the plate $\Omega_{m}$ transforming the strain energy caused by the dynamic deformation, Figure 1(b), into electric energy.

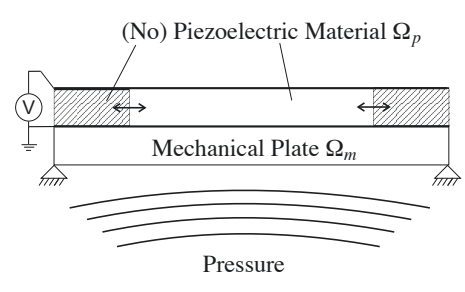

(a) Schema

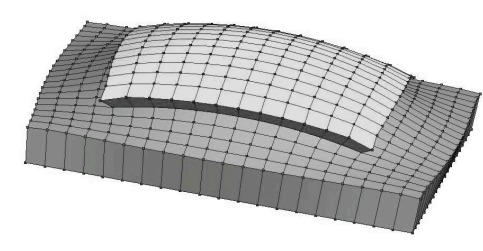

(b) Simulation

Figure 1: The plate $\Omega_{m}$ with support on the edge with the attached layer of piezoelectric material $\Omega_{p}$. The (sinusoidal) pressure deformes the system resulting in a voltage between the electrodes on both sides of $\Omega_{p}$. In Figure 1 (b) the heights of $\Omega_{m} 100 \mu \mathrm{m}$ and $50 \mu \mathrm{m}$ for $\Omega_{p}$ are scaled by factor of 100 . 
The quadratic aluminum plate $\Omega_{m}$ has a length of $50 \mathrm{~mm}$ and a thickness of $100 \mu \mathrm{m}$. The piezoelectric ceramic layer $\Omega_{p}(\mathrm{PZT}-5 \mathrm{~A})$ is $50 \mu \mathrm{m}$ thick (this rather thin materials have been used in a similar project, see the note below). There are electrodes on the free side of $\Omega_{p}$ and between $\Omega_{p}$ and $\Omega_{m}$. Their mechanical properties (stiffness) are neglected. We also assume an ideal connection between the two materials. Replacing the mechanical layer with a composite glue/plate layer would not change the introduced piezoelectric-mechanical coupled system. The presented optimization method is independent on the actual model properties (dimensions, thickness, material) as it provides for each specific model a specific optimal solution.

The material law describing the linear piezoelectric effect for $\Omega_{p}$ is given by

$$
\begin{aligned}
{[\boldsymbol{\sigma}] } & =\left[\boldsymbol{c}_{0}^{E}\right][\boldsymbol{S}]-\left[\boldsymbol{e}_{0}\right]^{T} \boldsymbol{E}, \\
\boldsymbol{D} & =\left[\boldsymbol{e}_{0}\right][\boldsymbol{S}]+\left[\boldsymbol{\varepsilon}_{0}^{S}\right] \boldsymbol{E} .
\end{aligned}
$$

The mechanical plate $\Omega_{m}$ supporting the piezoelectric layer is described by Hooke's law as

$$
[\boldsymbol{\sigma}]=\left[\boldsymbol{c}_{m}\right][\boldsymbol{S}] .
$$

The quantities are: Electric field intensity $\boldsymbol{E}$, electric displacement field $\boldsymbol{D}$, Cauchy stress tensor $[\boldsymbol{\sigma}]$, linear strain tensor $[S]$ and the material tensors of elastic moduli $\left[\boldsymbol{c}_{0}^{E}\right]$ and $\left[\boldsymbol{c}_{m}\right]$, dielectric permittivities $\left[\boldsymbol{\varepsilon}_{0}^{S}\right]$ and piezoelectric moduli $\left[\boldsymbol{e}_{0}\right]$.

The complete strong and weak formulation of the model above resulting in the finite element solution is given in (Wein et al, 2008a), a general description of multiphysics simulation can be found in (Kaltenbacher, 2007).

\section{Applying SIMP Optimization}

The SIMP method is an interpolating topology optimization method which has proved to be very efficient for mechanical problems (especially the compliance problem) but has been also applied successfully to other applications. The standard textbook (Bendsøe and Sigmund, 2003) has a strong focus on SIMP with various aspects and applications.

One of the earliest application of topology optimization to piezoelectric material is (Silva and Kikuchi, 1999) whereas (Kögl and Silva, 2005) explicitly refer to the SIMP method.

The idea is to introduce the design variable $\rho$ (pseudo density), modifying the material properties in such a way that a local value 1 for $\rho(x)$ with $x \in \Omega_{\text {opt }}$ represents material and a small value $\rho_{\min }$ represents void material (e.g. air). The interpretation of void material in this context is discussed in (Wein et al, 2008a). $\rho$ has a physical interpretation only for these two extremal values, nevertheless has the continuous approach advantages compared to an integer programming problem where only the limit values are possible.

The design vector $\boldsymbol{\rho}=\left(\rho_{1} \ldots \rho_{N_{e}}\right)^{T}$ with $\rho_{e} \in\left[\rho_{\min }: 1\right]$ shall be piecewise constant within the $N_{e}$ finite elements of the design domain.

We now introduce the notation $\widetilde{(\cdot)}$ for elements modified by the design parameter. Limiting the optimization domain to the piezoelectric layer, the following material parameters are formed by applying the design variable: $\left[\widetilde{\boldsymbol{c}}_{e}^{E}\right]=\mu_{c}\left(\rho_{e}\right)\left[\boldsymbol{c}_{0}^{E}\right]$, $\widetilde{\rho}_{e}^{\mathrm{m}}=\mu_{m}\left(\rho_{e}\right) \rho^{\mathrm{m}},\left[\widetilde{\boldsymbol{e}}_{e}\right]=\mu_{e}\left(\rho_{e}\right)\left[\boldsymbol{e}_{0}\right]$ and $\left[\widetilde{\boldsymbol{\varepsilon}}_{e}^{S}\right]=\mu_{\varepsilon}\left(\rho_{e}\right)\left[\boldsymbol{\varepsilon}_{0}^{S}\right]$. In contrast to common SIMP optimization we set all interpolation functions $\mu$ to the identity function $\mu_{(\cdot)}\left(\rho_{e}\right)=\rho_{e}$.

Applying the finite element method with the new ersatz material properties, the piecewise constant $\rho_{e}$ can be written in front of the bilinear forms. The components of the local mechanical stiffness matrix are given as:

$$
\widetilde{k}_{p q}^{u u}=\int_{\Omega_{e}}\left(\mathscr{B}_{p}^{u}\right)^{T}\left[\tilde{c}_{e}^{E}\right] \mathscr{B}_{q}^{u} d \Omega=\mu_{c}\left(\rho_{e}\right) \int_{\Omega_{e}}\left(\mathscr{B}_{p}^{u}\right)^{T}\left[\boldsymbol{c}_{0}^{E}\right] \mathscr{B}_{q}^{u} d \Omega=\rho_{e} k_{p q}^{u u}
$$

Hence the element wise SIMP design variable $\rho_{e}$ multiplies the local mechanical stiffness matrix as $\widetilde{\boldsymbol{K}}_{u u}^{e}=\mu_{c}\left(\rho_{e}\right) \boldsymbol{K}_{u u}^{e}$, similar for the mass matrix $\widetilde{\boldsymbol{M}}_{u u}^{e}=\mu_{m}\left(\rho_{e}\right) \boldsymbol{M}_{u u}^{e}$, the coupling matrix $\widetilde{\boldsymbol{K}}_{u \phi}^{e}=\mu_{e}\left(\rho_{e}\right) \boldsymbol{K}_{u \phi}^{e}$ and the permittivity matrix $\widetilde{\boldsymbol{K}}_{\phi \phi}^{e}=$ $\mu_{\varepsilon}\left(\rho_{e}\right) \boldsymbol{K}_{\phi \phi}^{e}$

Assuming a sinusoidal single-frequency excitation, a Fourier transformation of the system solves the steady state solution in the complex domain with imaginary unit $j$. Damping is introduced by the Rayleigh model with

$$
\boldsymbol{S}_{u u}^{e}(\omega)=\boldsymbol{K}_{u u}^{e}+j \omega \boldsymbol{C}_{u u}^{e}-\omega^{2} \boldsymbol{M}_{u u}^{e} ; \boldsymbol{C}_{u u}^{e}=\alpha_{K} \boldsymbol{K}_{u u}^{e}+\alpha_{M} \boldsymbol{M}_{u u}^{e}
$$

The global system of the finite element simulation is now written as

$$
\left(\begin{array}{ccc}
\boldsymbol{S}_{u_{m} u_{m}}(\omega) & \widetilde{\boldsymbol{S}}_{u_{m} u_{p}}(\omega, \boldsymbol{\rho}) & \mathbf{0} \\
\widetilde{\boldsymbol{S}}_{u_{m} u_{p}}^{T}(\omega, \boldsymbol{\rho}) & \widetilde{\boldsymbol{S}}_{u_{p} u_{p}}(\omega, \boldsymbol{\rho}) & \widetilde{\boldsymbol{K}}_{u_{p} \phi}(\boldsymbol{\rho}) \\
\mathbf{0} & \widetilde{\boldsymbol{K}}_{u_{p} \phi}^{T}(\boldsymbol{\rho}) & \widetilde{\boldsymbol{K}}_{\phi \phi}(\boldsymbol{\rho})
\end{array}\right)\left(\begin{array}{c}
\boldsymbol{u}_{m}(\omega, \boldsymbol{\rho}) \\
\boldsymbol{u}_{p}(\omega, \boldsymbol{\rho}) \\
\boldsymbol{\phi}(\omega, \boldsymbol{\rho})
\end{array}\right)=\left(\begin{array}{c}
\boldsymbol{f}_{m}(\omega) \\
\mathbf{0} \\
\mathbf{0}
\end{array}\right)
$$


or in shortened form as

$$
\widehat{\tilde{\boldsymbol{S}}}(\omega, \boldsymbol{\rho}) \widehat{\boldsymbol{u}}(\omega, \boldsymbol{\rho})=\widehat{\boldsymbol{f}}(\omega) \text { or } \widehat{\tilde{\boldsymbol{S}}} \widehat{\boldsymbol{u}}=\widehat{\boldsymbol{f}} .
$$

Note that with the presented form any topology of the piezoelectric layer can be expressed on the same finite element mesh. $\widehat{\boldsymbol{u}}$ does not explicitly depend on $\rho$ but implicitly. We ignore this difference for the sake of a clearer formulation of the objective functions.

\section{Objective Functions}

A significant portion of work on topology optimization of piezoelectric material is published in cooperation with E.C.M Silva. They all share an objective function that is based on the so called mean transduction in combination with a minimization of the mechanical stiffness. This also holds for the work on topology optimization (Nakasone et al, 2008).

The mean transduction gives a measure for the coupling between the electrostatic and mechanical field, or as formulated in (Kögl and Silva, 2005) "... the conversion of electric into elastic energy and vice versa". It is based on two load cases and choosing the load cases properly, the maximization of the transduction corresponds to the maximization of the displacement with respect to the load cases. Being a nodal force and a charge, these load cases result in our coupled model in vanishing piezoelectric material for a maximal transduction (Wein et al, 2008a) and is such not appropriate for the given model.

Our analysis of the mean transduction also showed, that for a nodal force excitation the given model shows the highest electric potential for a vanishing piezoelectric layer. This is due to the non-physical excitation and the linear model. A vanishing ceramic layer represents the least stiffness and hence the largest bending at the point of excitation. This results in a maximal (local) strain and hence a maximal electric potential. This shows that the measure of the electric potential as objective function shall be considered with care (note that we assume a pressure excitation in the present work).

In (Bin Zheng and Gea, 2008) the objective function is the efficiency factor (note that only a static model is considered and the properties below are real valued)

$$
\eta=\frac{W_{\text {elec }}}{W_{\text {total }}}
$$

with $W_{\text {elec }}=\frac{1}{2} \boldsymbol{\phi}^{T} \widetilde{\boldsymbol{K}}_{\phi \phi} \boldsymbol{\phi}$ and $W_{\text {total }}=W_{\text {elec }}+W_{\text {mech }}=\frac{1}{2} \boldsymbol{\phi}^{T} \widetilde{\boldsymbol{K}}_{\phi \phi} \boldsymbol{\phi}+\frac{1}{2} \boldsymbol{u}^{T} \widetilde{\boldsymbol{K}}_{\text {uu }} \boldsymbol{u}$.

It has been found and described in (Weller, 2009) that maximizing $\eta$ involves the minimization of $W_{\text {mech }}$, also known as maximization of the stiffness. The stiffness maximization is the usual application of the SIMP method but for the given objective Weller showed that the optimizer may sacrifice $W_{\text {elec }}$ for the sake of a large $W_{\text {mech }}$ (stiff material). See also Figure 2(c).

As we may assume in typical energy harvesting applications the ambient energy to be sufficiently large, we choose the electric energy and the potential as our optimization functions.

An objective function $\mathscr{J}(\widehat{\boldsymbol{u}}(\boldsymbol{\rho}, \omega))=J$ needs the property to map to a real value $J: \rightarrow \mathbb{R}$ to be comparable. Hence we have the optimization problem $\max _{\rho} J$ with the implicit condition $\widehat{\tilde{\boldsymbol{S}}} \widehat{\boldsymbol{u}}=\widehat{\boldsymbol{f}}$ and the box constraints $\rho_{e} \in\left[\rho_{\min }: 1\right]$. $J=J_{\phi}=\phi^{T} \boldsymbol{L} \boldsymbol{\phi}^{*}$ or $J=J_{\text {elec }}=\boldsymbol{\phi}^{T} \widetilde{\boldsymbol{K}}_{\phi \phi} \boldsymbol{\phi}^{*}$ with $\boldsymbol{\phi}^{*}$ a vector with complex conjugate electric potentials of the solution vector. The matrix $L$ has only entries on its diagonal. The diagonal entries corresponding to the potential nodes on the free electrode are set to 1 otherwise they are set to 0 . Hence we can also write $J_{\phi}=\widehat{\boldsymbol{u}}^{T} \boldsymbol{L} \widehat{\boldsymbol{u}}^{*}$ what corresponds to the form we used in (Wein et al, 2008a) where further details can be found. As we want to maximize the potential it does not matter, that the objective function actually represents the squared potential. The piezoelectric model describes a inhomogeneous potential field that is averaged by the equipotential layer formulation. Note, that for this averaging also the potential nodes corresponding to piezoelectric areas set to void by $\rho_{\min }$ are considered. But due to low permittivity the contribution is correspondingly low.

The energy formulation $J_{\text {elec }}=\phi^{T} \widetilde{\boldsymbol{K}}_{\phi \phi}(\boldsymbol{\rho}) \boldsymbol{\phi}^{*}$ can be read as an assembly (or sum if the dimensions are adjusted) of the element matrices and solutions $J_{\text {elec }}=\sum_{N_{e}} \phi_{e}^{T} \mu\left(\rho_{e}\right) \boldsymbol{K}_{\phi \phi}^{0} \boldsymbol{\phi}_{e}^{*}$. For elements with a pseudo density $\rho_{e}$ close to $\rho_{\min }$ the contribution is negligible.

A key feature of the SIMP method is, that due to the adjoint method it is relatively easy to find the partial derivatives of the objective function with respect to the design variables. This is called sensitivity analysis and for $J=J_{\phi}$ and $J=J_{\text {elec }}$ the gradient is given as

$$
\frac{\partial J}{\partial \rho_{e}}=2 \operatorname{Re}\left\{\boldsymbol{\lambda}^{T} \frac{\partial \widehat{\widetilde{\boldsymbol{S}}}}{\partial \rho_{e}} \boldsymbol{\phi}\right\}
$$

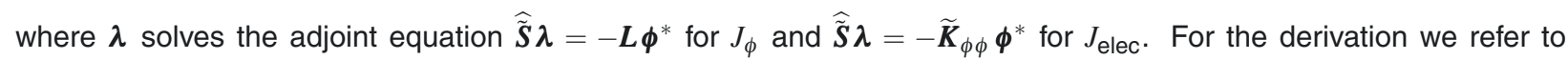
(Jensen, 2007). 
Having the objective function, its derivative and (for the moment) no further constraint function, an external nonlinear optimizer can solve the problem. State of the art in topology optimization is the Method of Moving Asymptotes (MMA) by Krister Svanberg. We use the fast and globalized implementation SCPIP (Zillober, 2002) via our interface C++SCPIP (Wein, 2007).

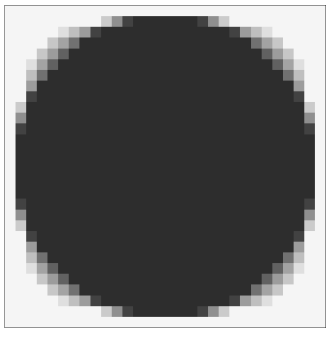

(a) Potential

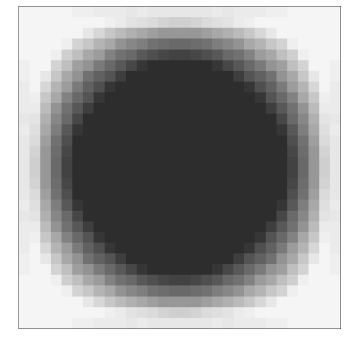

(b) Electric Energy

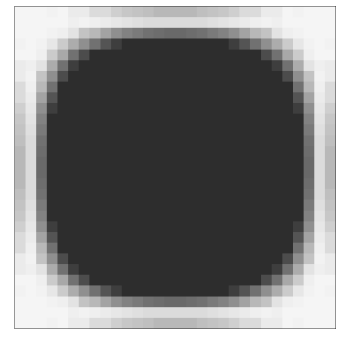

(c) Energy Conversion

Figure 2: For the model introduced in Figure 1(a) we apply (for the static case) three different objective functions. The optimal topology/ material distribution of the piezoelectric material $\Omega_{p}$ is displayed. Black represents material, white no/void material and grey values unphysical "intermediate" material. The square underlying material plate $\Omega_{m}$ receiving the pressure excitation is not optimized. Figure 2(a) maximizes for $\phi$ on the free electrode, Figure 2(b) integrates within the ceramic $\phi^{T} \widetilde{\boldsymbol{K}}_{\phi \phi}(\boldsymbol{\rho}) \boldsymbol{\phi}$ and Figure 2(c) maximizes the energy efficiency factor by increasing the mechanical stiffness.

\section{Results}

We have a mono-frequent system, driven by a harmonic pressure excitation. To evaluate our proposed method we perform the optimization excitation frequencies from $10 \mathrm{~Hz}$ to $2000 \mathrm{~Hz}$ with a fine stepping. To handle the computational effort the optimizer was limited to 200 iterations per frequency.

With the amount and location of piezoelectric material as design variable, one could expect that the optimizer tries to build a system with a resonance frequency as close as possible to the excitation frequency. What makes actually the difference between the objective functions for potential and electric energy, are vibrational patterns corresponding to a mechanical resonance but with compensating positive and negative strains (see our reference publication for more details). For this case the integral electric energy might be high but electric potential between the electrodes is low.

Common SIMP optimization is based on a volume constraint limiting the maximal allowed fraction of material. This is together with other techniques a common way to ensure that the design variable pseudo density is driven toward 1 or some small $\rho_{\min }$ for an interpretation of material or no/void material. Intermediate values, e.g. 0.5 ("half material") have no physical interpretation. In mechanics this could be seen as a corresponding local height of the layer at the element (the so called variable thickness sheet problem) but in the piezoelectric case this is possible.

Having no engineering reason for a volume constraint we therefore have to validate the resulting pseudo density distribution. This is called in SIMP terminology as greyness which is given (normalized to 1) as

$$
g_{\text {grey }}=\int_{\Omega} 4 *(1-\rho) \rho d x
$$

Greyness values below 0.1 can be considered as sufficient distinct $0-1$ result. For typical elasticity problems (maximization of the stiffness) the greyness cannot be used as a constrained as due to the concave structure the optimizer tends to be locked by local optima. For the present problem, do we observe greyness problem mostly when optimizing for $W_{\text {elec }}$ (see Figure $3(\mathrm{~d})$ and $4(\mathrm{k})$ ). Adding a greyness constraint works in principle but in several cases below the first resonance frequency (see Figure $3(d)$ ). The impact to the resulting objective value is for most frequencies negligible, see 3(b).

\section{Conclusions}

We have presented a method to construct an energy harvester that converts the harmonic deflection of a thin plate by the help of piezoelectric material into electric energy. By the help of topology optimization the objective functions electric potential and electric energy can be increased significantly.

We have applied the method to several sinusoidal excitations. This parameter study revealed problems with respect to robustness $3(\mathrm{a})$ and $3(\mathrm{~b})$ and greyness $3(\mathrm{~d})$. It shall be mentioned that due to the complexity of highly nonlinear 


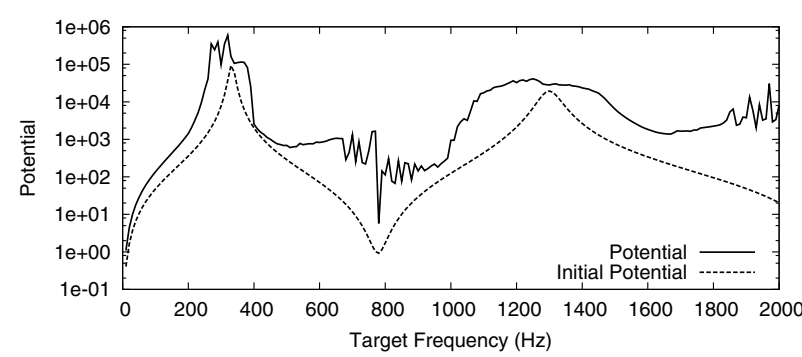

(a) Maximize Potential

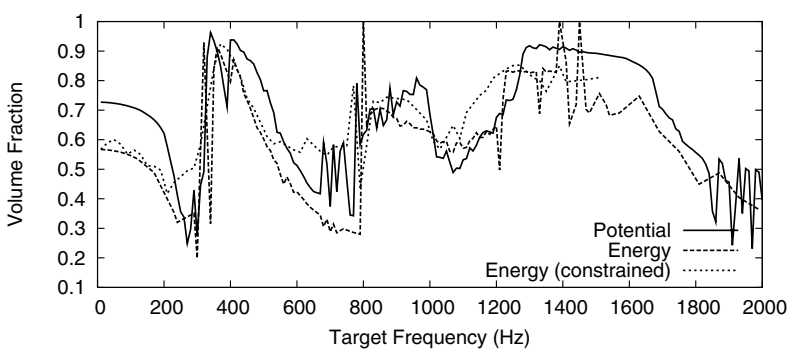

(c) Volume

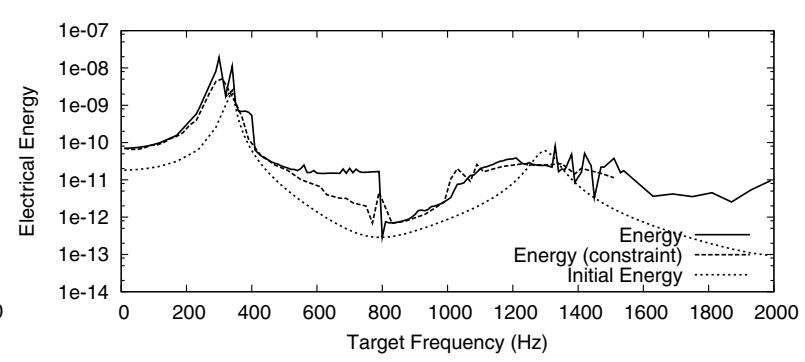

(b) Maximize Electric Energy

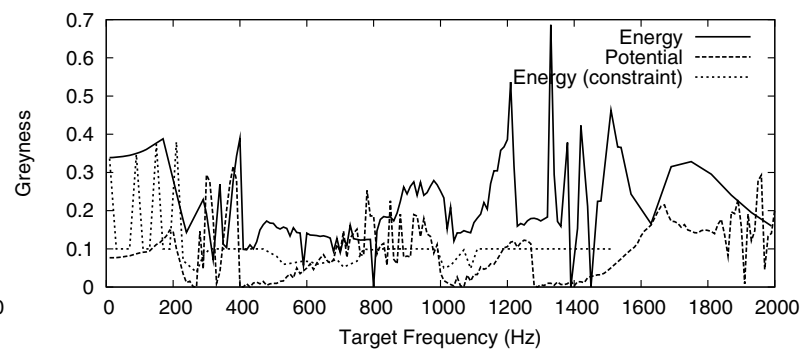

(d) Greyness

Figure 3: We perform several optimizations for target frequencies from $10 \mathrm{~Hz}$ to $2000 \mathrm{~Hz}$. In Figure $3\left(\right.$ a) for $J_{\phi}=\boldsymbol{\phi}^{T} \boldsymbol{L} \boldsymbol{\phi}^{*}$ and in Figure 3(b) for $J_{\text {elec }}=\boldsymbol{\phi}^{T} \widetilde{\boldsymbol{K}}_{\phi \phi}(\boldsymbol{\rho}) \boldsymbol{\phi}^{*}$ (one time the greyness constrained applied). For reference the value for the initial full piezoelectric plate is given. In Figure 3(c) we see the impact of the amount of material to adjust resonance frequencies. In Figure 3(d) we see that for the energy objective function such a constraint is necessary but also that it works not reliable. Due to the linear model and assumed damping values are the magnitudes of the objective function not realistic what has no impact to the topology.

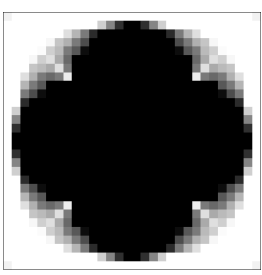

(a) $200 \mathrm{~Hz} J_{\phi}$

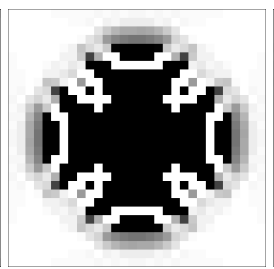

(b) $200 \mathrm{~Hz} J_{\text {elec }}$

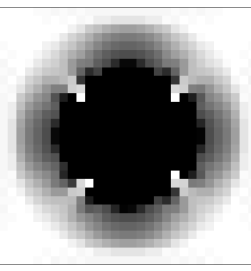

(c) $210 \mathrm{~Hz} J_{\text {elec }}+g_{\text {grey }}$

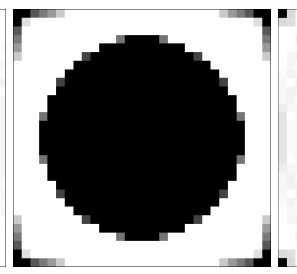

(d) $600 \mathrm{~Hz} J_{\phi}$

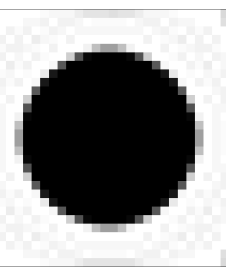

(e) $620 \mathrm{~Hz} J_{\text {elec }}$

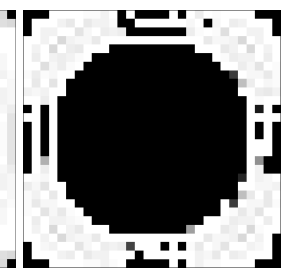

(f) $610 \mathrm{~Hz} J_{\text {elec }}+g_{\text {grey }}$

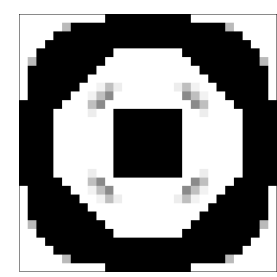

(g) $1100 \mathrm{~Hz} J_{\phi}$

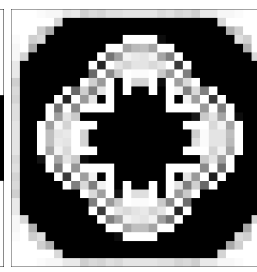

(h) $1110 \mathrm{~Hz} J_{\text {elec }}$

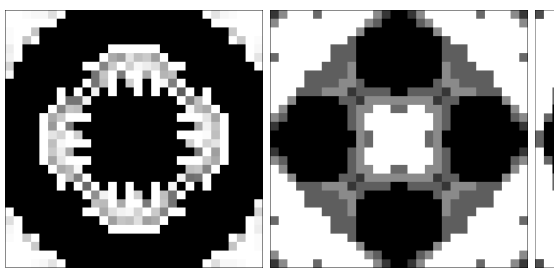

(i) $1110 \mathrm{~Hz} J_{\text {elec }}+g_{\text {grey }}$ (j) $1950 \mathrm{~Hz} J_{\phi}$

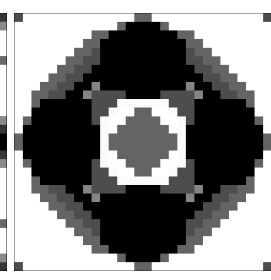

(k) $1850 \mathrm{~Hz} J_{\text {elec }}$

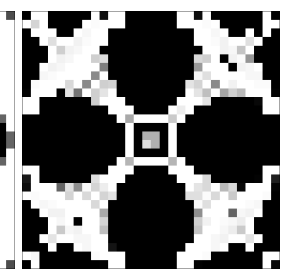

(l) $1850 \mathrm{~Hz} J_{\text {elec }}+g_{\text {grey }}$

Figure 4: Some sample optimization results from the parameter studies in Figures 3(a) and 3(b). See Figure 2(a) for an explanation of the color scale.

topology optimization problems it is common practice to perform manual tuning of optimization parameters for a given problem. On the other hand is improved robustness a necessary feature for multiple-frequency broadband optimization as a next step.

The focus of this work is the presented method. The feasibility of the resulting topologies with respect to constructibility respectively manufacturability has not been discussed. A modification of the model in Figure 1 (a) with respect to material, thickness and glue layer might ease this issue. As mentioned in (Bendsøe and Sigmund, 2003) does topology optimization provide guidelines and hints for designers and not the final blueprint.

An important finding in (Wein et al, 2008b) has been the ability of the optimizer to create vibrational patterns that resonate by cancelling positive and negative strains within the piezoelectric layer. This effect could be only partially 
repeated.

\section{References}

Anton S, Sodano H (2007) A review of power harvesting using piezoelectric materials (2003-2006). Smart Mater Struct 16(3)

Bendsøe M, Sigmund O (2003) Topology Optimization: Theory, Method and Applications, 2nd edn. Springer Verlag

Bin Zheng CJC, Gea HC (2008) Topology optimization of energy harvesting devices using piezoelectric materials. Struct Multidisc Optim 38(1):17-23

Jensen JS (2007) A note on sensitivity analysis of linear dynamic systems with harmonic excitation, handout at DCAMM advanced school June 20-26, 2007 at DTU in Lyngby, Denmark.

Kaltenbacher M (2007) Numerical Simulation of Mechatronic Sensors and Actuators, 2nd edn. Springer Verlag

Kögl M, Silva ECN (2005) Topology optimization of smart structures: design of piezoelectric plate and shell actuators. Smart Mater Struct 14(2):387-399

Nakasone P, Kiyono C, Silva ECN (2008) Design of piezoelectric sensors, actuators, and energy harvesting devices using topology optimization. In: Proceedings of SPIE, SPIE, vol 6932, p 69322W

Silva ECN, Kikuchi N (1999) Design of piezoelectric transducers using topology optimization. Smart Mater Struct 8(3):350-364

Wein F (2007) C++SCPIP, a C++ wrapper for SCPIP. Online, http://cppmath.sourceforge.net

Wein F, Kaltenbacher M, Kaltenbacher B, Leugering G, Bänsch E, Schury F (2008a) Topology Optimization of Piezoelectric Layers Using the SIMP Method, submitted for review to Struct Multidisc Optim

Wein F, Kaltenbacher M, Leugering G, Bänsch E, Schury F (2008b) Topology optimization of a piezoelectric-mechanical actuator with single- and multiple-frequency excitation, submitted for review to COMPEL

Weller E (2009) Topology optimization of a piezoelectric energy harvester. Master's thesis, University of ErlangenNuremberg, Germany, in German

Zillober C (2002) SCPIP - an efficient software tool for the solution of structural optimization problems. Struct MultidisC Optim 24(5):362-371 